\title{
BESO APPROACH TO TOPOLOGY OPTIMIZATION OF GAN PHONONIC CRYSTALS
}

\author{
Luca D’Alessandro ${ }^{1,2^{*}}$, Bichoy Bahr ${ }^{2}$, Luca Daniel ${ }^{3}$, Dana Weinstein ${ }^{4}$, Raffaele Ardito ${ }^{1}$ \\ ${ }^{1}$ Dept. of Civil and Environmental Engineering, Politecnico di Milano \\ Milan, Italy \\ e-mail: \{luca.dalessandro, raffaele.ardito\}@polimi.it
}

${ }^{2}$ Dept. of Electrical Engineering and Computer Science, Massachusetts Institute of Technology

Cambridge (MA), USA

\{lucadale, bichoy\}@mit.edu

${ }^{3}$ Research Laboratory of Electronics, Massachusetts Institute of Technology

Cambridge (MA), USA

luca@mit.edu

${ }^{4}$ Dept. of Electric and Computer Engineering, Purdue University

West Lafayette (IN), USA

danaw@purdue.edu

Keywords: Phononic Crystals, Resonators, Acoustic waves, Bandgap, Topology optimization, Micromechanics

\begin{abstract}
The use of Phononic Crystals (PnC) in suspended structures and microstructures, such as plates and slabs, has gained a lot of attention in the past years for the wide range of feasible applications (acoustic waveguides, acoustic insulation, acoustic cloaking) and for the easy fabrication technique. Since the performance of the device is related to the band of frequencies reflected by the PnC and since this band (called bandgap) depends on the geometric and material properties of the fundamental unit cell of the PnC, a useful tool for the design of those structures is topology optimization. This paper is focused on a novel and fast engineering use of Bidirectional Evolutionary Structural Optimization for the definition of the optimal hole configuration in air-solid PnC. The technique adopted finds the optimal shape of the hole in less than 20 iterations, and it is easy implemented in a 2D plane strain Matlab finite element solver.
\end{abstract}




\section{INTRODUCTION}

Phononic Crystals (PnCs) gained lots of attention over the past several years, owing to their versatility and wide spectrum of applications: acoustic isolation, noise suppression, vibration attenuation, acoustic waveguides, acoustic super-lenses, negative refraction and acoustic cloaking [1],[2].

This paper is focused on the use of PnCs in "unreleased" resonators [3], which overcome many challenges faced by traditional MEMS resonators, including non-linearity, power handling, residual stresses and yield [4]. PnCs are represented by periodic media, which exhibit bandgaps, i.e. bounded frequency regions for which the propagation of sound waves through the crystal is impeded. The central frequency and the size of the bandgap basically depend on the materials employed in PnCs and on the shape of the unit cell.

Most of the abovementioned applications will significantly benefit from PnCs with larger bandgap. For this reason, it is highly desirable to optimize the shape of a $\mathrm{PnC}$ unit cell, in order to obtain the largest bandgap while complying with manufacturability constraints.

The present paper aims at the achievement of the best shape for the gallium nitride (GaN) solid-hole PnC unit cell, producing the largest possible phononic bandgap around a given center frequency.

The procedure starts from a given geometry, endowed with a certain bandgap, and proceeds with an automatic shape optimization algorithm in order to evolve this geometry into an optimal one for maximum bandgap around the requested central frequency. Several research groups have treated the topological optimization of PnCs, e.g. by using the SIMP approach and the MMA algorithm [5], genetic algorithm [6]-[8]. In this work, we consider the application of Bidirectional Evolutionary Structural Optimization (BESO) that was previously applied for Photonic Crystals [9] and is now used for the first time in the solid-hole PnCs with an engineering perspective. The algorithm is able to modify the shape by adding and removing elements, using the FEM-based sensitivity analysis for the acoustic wave frequency.

The proposed optimization algorithm can successfully obtain the optimal topology in less than 20 iterations: the optimization process involves a 500\% gain with respect to the initial bandgap.

The optimal dimensionless bandgap depends only on the hole's shape and on the ratio between elastic constants of the material. Attention is paid to the robustness of the achieved solution, in view of the construction tolerances, which might alter the optimality of the real device.

\section{FINITE ELEMENT PROBLEM FORMULATION}

PnC structures are periodic structures generally composed of two or more elastic material phases, solid or fluid. The theoretical framework is wave propagation in elastic inhomogeneous media, and it is governed by the following elasto-dynamic equations [10], [11]:

$$
\begin{aligned}
& \rho \frac{\partial^{2} u_{x}}{\partial t^{2}}=\frac{\partial}{\partial x}\left(\lambda \nabla\left[u_{x}, u_{y}, u_{z}\right]^{T}\right)+\nabla\left(\mu\left(\nabla u_{x}+\frac{\partial\left[u_{x}, u_{y}, u_{z}\right]^{T}}{\partial x}\right)\right) \\
& \rho \frac{\partial^{2} u_{y}}{\partial t^{2}}=\frac{\partial}{\partial y}\left(\lambda \nabla\left[u_{x}, u_{y}, u_{z}\right]^{T}\right)+\nabla\left(\mu\left(\nabla u_{y}+\frac{\partial\left[u_{x}, u_{y}, u_{z}\right]^{T}}{\partial y}\right)\right) \\
& \rho \frac{\partial^{2} u_{z}}{\partial t^{2}}=\frac{\partial}{\partial z}\left(\lambda \nabla\left[u_{x}, u_{y}, u_{z}\right]^{T}\right)+\nabla\left(\mu\left(\nabla u_{x}+\frac{\partial\left[u_{x}, u_{y}, u_{z}\right]^{T}}{\partial z}\right)\right)
\end{aligned}
$$


where $\rho$ is the material density, $\lambda$ and $\mu$ are Lamé's coefficients, $u_{x}, u_{y}, u_{z}$ the cartesian components of the displacement vector.

The case study analyzed in this paper is referred to PnC slabs composed of air-solid phase materials, it is possible to assume some simplifications such that:

- the material parameters $\rho, \lambda$ and $\mu$ depend only on the $x$ and $y$ (in-plane) coordinates: $\rho=\rho(x, y), \lambda=\lambda(x, y), \mu=\mu(x, y)$;

- being the thickness of the slab small compared to the in-plane dimensions, plane strain hypothesis can be exploited: $\partial\left[u_{x}, u_{y}, u_{z}\right]^{T} / \partial z=\mathbf{0}, u_{z}=0$.

Due to the previous hypotheses, the elasto-dynamic problem (1) can be formulated as:

$$
\begin{aligned}
& \rho \frac{\partial^{2} u_{x}}{\partial t^{2}}=\frac{\partial}{\partial x}\left((2 \mu+\lambda) \frac{\partial u_{x}}{\partial x}+\lambda \frac{\partial u_{y}}{\partial y}\right)+\frac{\partial}{\partial y}\left(\mu\left(\frac{\partial u_{x}}{\partial y}+\frac{\partial u_{y}}{\partial x}\right)\right) \\
& \rho \frac{\partial^{2} u_{y}}{\partial t^{2}}=\frac{\partial}{\partial x}\left(\mu\left(\frac{\partial u_{y}}{\partial x}+\frac{\partial u_{x}}{\partial y}\right)\right)+\frac{\partial}{\partial y}\left((2 \mu+\lambda) \frac{\partial u_{y}}{\partial y}+\lambda \frac{\partial u_{x}}{\partial x}\right)
\end{aligned}
$$

where the only unknowns are $u_{x}=u_{x}(\mathbf{r}, \mathbf{k}, t), u_{y}=u_{y}(\mathbf{r}, \mathbf{k}, t)$, where $\mathbf{r}=[x, y]^{T}$ is the vector of coordinates in the plane, $\mathbf{k}=\left[k_{x}, k_{y}\right]^{T}$ is the plane wave number vector, $t$ is the time.

In the frequency domain, the equations (2) become:

$$
\begin{aligned}
& -\rho u_{x} \omega^{2}=\frac{\partial}{\partial x}\left((2 \mu+\lambda) \frac{\partial u_{x}}{\partial x}+\lambda \frac{\partial u_{y}}{\partial y}\right)+\frac{\partial}{\partial y}\left(\mu\left(\frac{\partial u_{x}}{\partial y}+\frac{\partial u_{y}}{\partial x}\right)\right) \\
& -\rho u_{y} \omega^{2}=\frac{\partial}{\partial x}\left((2 \mu+\lambda) \frac{\partial u_{x}}{\partial x}+\lambda \frac{\partial u_{y}}{\partial y}\right)+\frac{\partial}{\partial y}\left(\mu\left(\frac{\partial u_{x}}{\partial y}+\frac{\partial u_{y}}{\partial x}\right)\right)
\end{aligned}
$$

where $\omega$ is the angular frequency, $u_{x}=u_{x}(\mathbf{r}, \mathbf{k}), u_{y}=u_{y}(\mathbf{r}, \mathbf{k})$ are no more function of time.

Due to the periodicity of the PnC, it's possible to study the elasto-dynamic problem referring to the single cell only, exploiting the Floquet-Bloch theorem [12]:

$$
\begin{aligned}
& u_{x}=u_{x}(\mathbf{r}, \mathbf{k})=u_{x}(\mathbf{r}) e^{i \mathbf{k r}} \\
& u_{y}=u_{y}(\mathbf{r}, \mathbf{k})=u_{y}(\mathbf{r}) e^{i \mathbf{k r}}
\end{aligned}
$$
tion:

In the literature, there are two approaches to introduce (4) in the problem under investiga-

- direct substitution in (3) and proceed to the weak formulation of the equations and the finite element (FE) discretization ([5],[10],[11]);

- first weak formulation (as usual for elasto-dynamic problems), then FE discretization, and at the end enforce the equations (4) as periodic boundary conditions ([13],[14],[15]).

Being the aim of this work the implementation of a topology optimization technique, it is more suitable to refer to the second approach since in this way the assembly of the general mass and stiffness matrices of the problem is not depending on the wave vector $\mathbf{k}$ and therefore it is done only once for each $\mathbf{k}$ vector.

It is possible to discretize the weak form of equations (3) into finite elements, being the inplane displacements expressed like: 


$$
\begin{aligned}
& u_{x}=u_{x}(\mathbf{r}, \mathbf{k})=\sum_{e l} \sum_{j=1}^{n} N_{j} u_{x j}^{e l} \\
& u_{y}=u_{y}(\mathbf{r}, \mathbf{k})=\sum_{e l} \sum_{j=1}^{n} N_{j} u_{y j}^{e l}
\end{aligned}
$$

where $e l$ is the index for the elements of the mesh, $j$ is the index for the nodes of each element, $N_{j}$ are the shape functions for the quadrilateral plane element: $j=1,2,3,4, u_{x j}^{e l}$ and $u_{y j}^{e l}$ are the components of the displacement of each node of an element.

The linear quadrilateral element has been chosen and, in particular, a square FE shape in order to build a regular grid of elements over the unit cell: in this way the algorithm of topological optimization is easily implemented, dealing with each element as a "pixel" that can be switched between fill and void. Being the aim of this work the optimization of a solid/air PnC the finite element formulation is such that the only elements that contribute to the matrices are the solid elements: the air domain is not considered.

The FE discretization (5) of equations (3) yields the governing FE governing equation:

$$
\begin{gathered}
\mathbf{u}^{T}(\mathbf{k}, \mathbf{r})\left(\mathbf{K}-\omega^{2} \mathbf{M}\right) \mathbf{u}(\mathbf{k}, \mathbf{r})=0 \\
\mathbf{u}(\mathbf{k}, \mathbf{r})=\left[u_{j x}(\mathbf{k}, \mathbf{r}), u_{j y}(\mathbf{k}, \mathbf{r}), \ldots \mathbf{j}=1,2 . . \mathrm{N}_{\text {nodes }}\right]^{T}
\end{gathered}
$$

where $\mathbf{K}$ and $\mathbf{M}$ are the assembled global stiffness and mass matrices that do not depend on $\mathbf{k}, \mathbf{u}(\mathbf{k}, \mathbf{r})$ is the global vector of unknowns containing in order the $x$ and $y$ components of each node displacement.

In order to formulate the complete FE problem, some suitable boundary conditions must be defined together with equations (6):

- free boundary condition is applied to the boundary elements of the solid domain in the interface with air domain;

- periodic boundary conditions are defined after reordering the nodes of the mesh as shown in Figure 1.

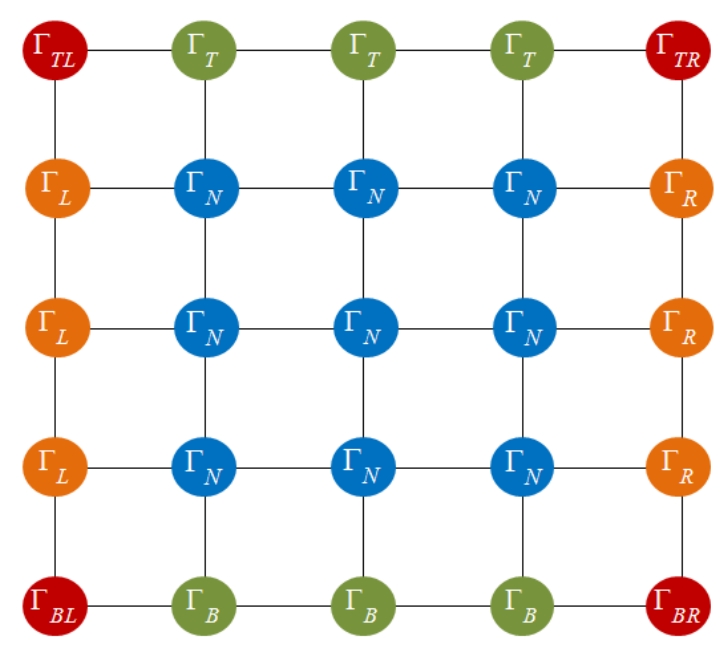

Figure 1: FE grid with nodes identified by color and type with respect to periodicity boundary conditions.

$\Gamma$ stands for boundary, $T$ for Top, $B$ for Bottom, $L$ for Left, $R$ for Right, , $N$ for Internal.

The nodes of different types identified with the same color are related by the bc. 
By means of this organization, it is possible to define the periodic boundary conditions in a matrix form:

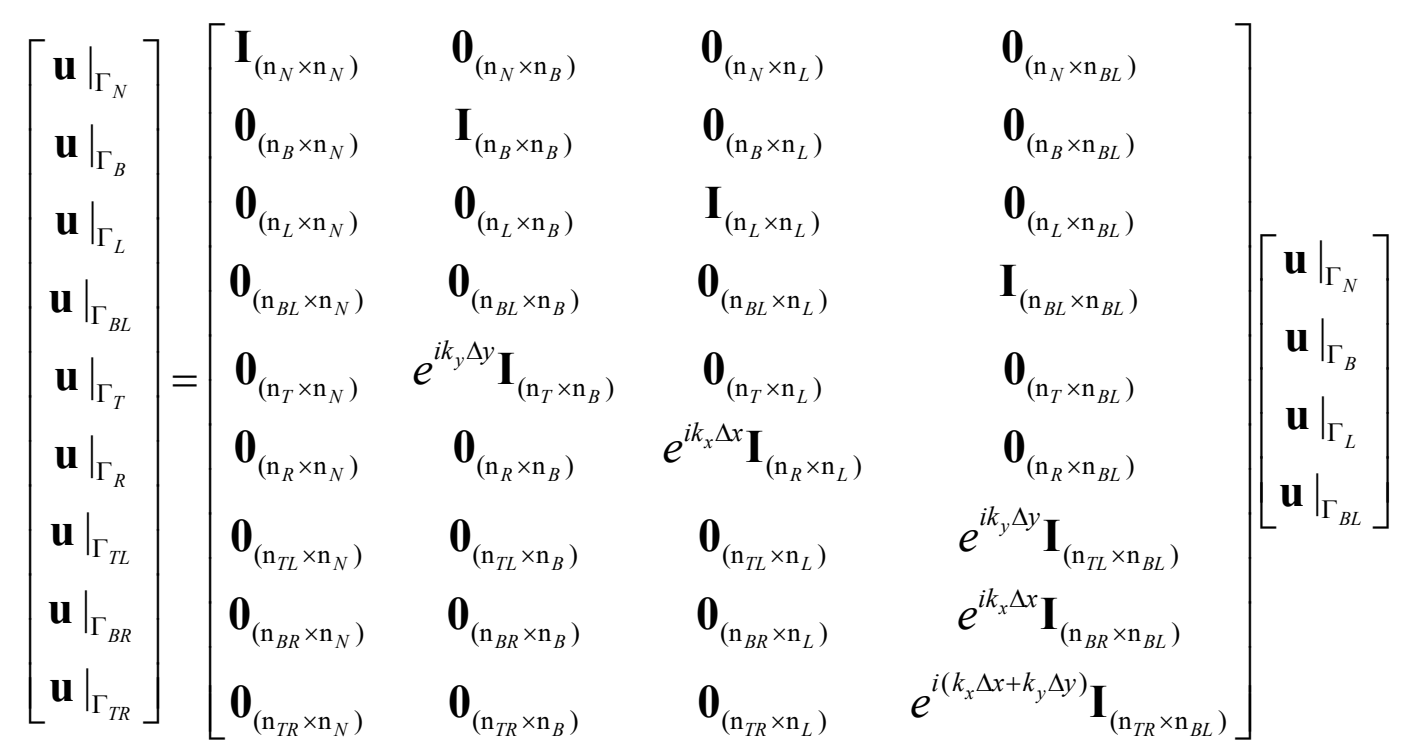

where the symbol $\mid$ denotes "on the boundary", and $\Delta x$ and $\Delta y$ denote the offset between the coordinates of the nodes in $\mathrm{x}$ and $\mathrm{y}$ direction, respectively.

Equation (7) can be rewritten as:

$$
\mathbf{u}(\mathbf{k}, \mathbf{r})=\mathbf{T}(\mathbf{k}, \mathbf{r}) \tilde{\mathbf{u}}(\mathbf{r})
$$

where $\tilde{\mathbf{u}}(\mathbf{r})=\left[\left.\mathbf{u}\right|_{\Gamma_{N}}{ }^{T},\left.\mathbf{u}\right|_{\Gamma_{B}}{ }^{T},\left.\mathbf{u}\right|_{\Gamma_{L}}{ }^{T},\left.\mathbf{u}\right|_{\Gamma_{B L}}{ }^{T}\right]^{T}$ that collects only independent displacements.

Substituting equation (8) in equation (6):

$$
\tilde{\mathbf{u}}^{T}(\mathbf{r}) \mathbf{T}^{H}(\mathbf{k}, \mathbf{r})\left(\mathbf{K}-\omega^{2} \mathbf{M}\right) \mathbf{T}(\mathbf{k}, \mathbf{r}) \tilde{\mathbf{u}}(\mathbf{r})=0
$$

where ${ }^{H}$ stands for Hermitian transpose.

The following problem matrices are Hermitian:

$$
\begin{aligned}
\tilde{\mathbf{K}} & =\mathbf{T}^{H}(\mathbf{k}, \mathbf{r})(\mathbf{K}) \mathbf{T}(\mathbf{k}, \mathbf{r}) \\
\tilde{\mathbf{M}} & =\mathbf{T}^{H}(\mathbf{k}, \mathbf{r})(\mathbf{M}) \mathbf{T}(\mathbf{k}, \mathbf{r})
\end{aligned}
$$

Introducing (10) in (9) one obtains:

$$
\tilde{\mathbf{u}}^{T}(\mathbf{r})\left(\tilde{\mathbf{K}}-\omega^{2} \tilde{\mathbf{M}}\right) \tilde{\mathbf{u}}(\mathbf{r})=0
$$

Equation (11) must be solved in order to find eigenvalues $\omega_{n}{ }^{2}$, eigenvectors $\tilde{\mathbf{u}}_{n}(\mathbf{r})$ and the associated dispersion relation at varying $\mathbf{k}$.

To obtain a good description of the dynamic behavior of the PnC for all the possible $\mathbf{k}$ vectors in the $\mathbf{k}$ space (plane), it is possible to choose a limited number of $\mathbf{k}$ vectors, discretizing the boundary of the first irreducible Brillouin zone [16]. In Figure 2, the rectangle in $\mathbf{k}$ space is the first Brillouin zone. Due to the square symmetry of the unit cell, it is possible to focus the attention only on the colored triangle (first irreducible Brillouin zone). It is claimed then from many authors that from the engineering point of view the maximum and minimum points of the bandgap can be determined only focusing the attention on the $\mathbf{k}$ vectors along the boundary of the First Irreducible Brillouin zone, following the path indicated in Figure 3. 


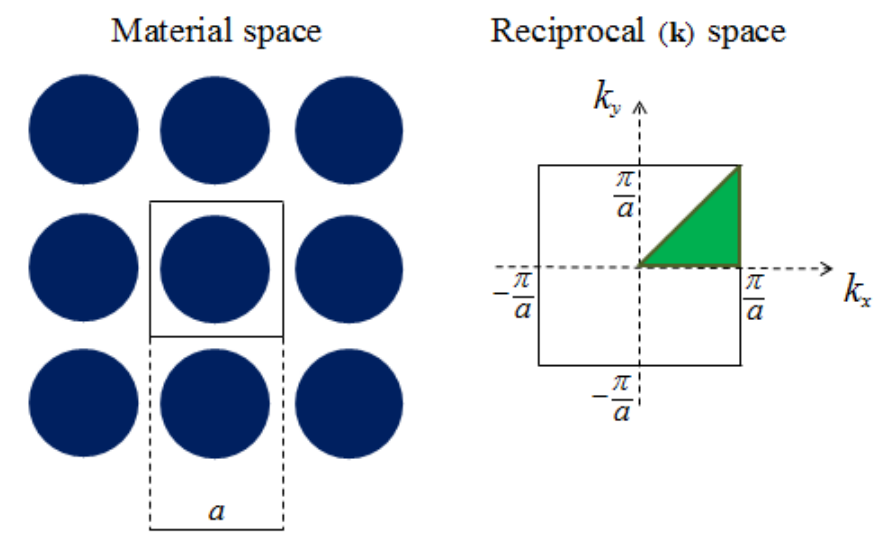

Figure 2: Material and reciprocal space, First Brillouin Zone.

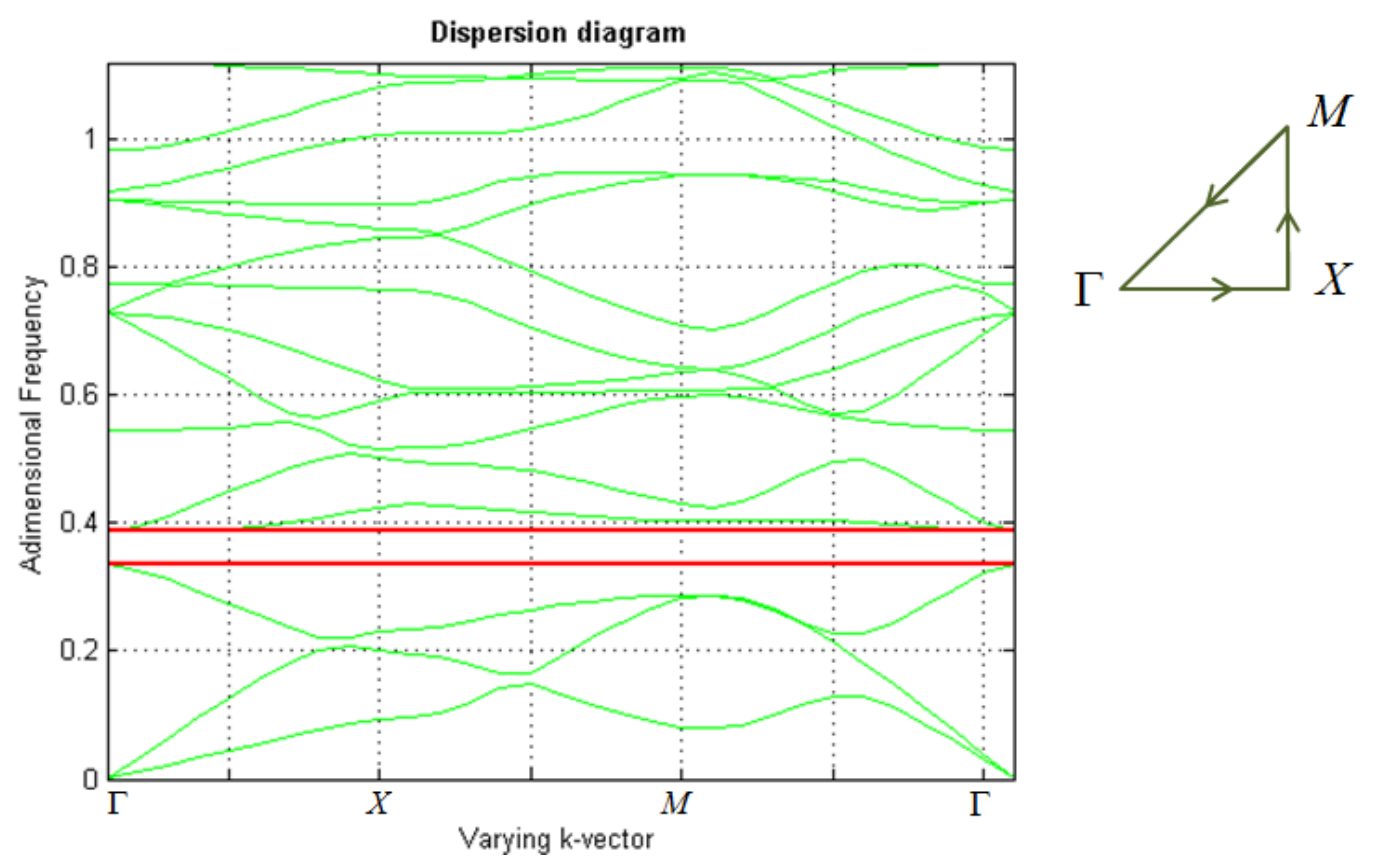

Figure 3: Dispersion Diagram for k-vector in the First Irreducible Brillouin Zone.

\section{OPTIMIZATION PROBLEM FORMULATION}

\subsection{Objective function}

The aim of this work is to find the optimal topology that maximizes an existing bandgap around a certain central frequency for solid/air PnC.

The objective function of this optimization is the bandgap width normalized with respect to the central frequency of the bandgap:

$$
\max _{\mathbf{\rho}}\left\{b g(\mathbf{\rho})=\frac{\Delta \omega^{2}(\mathbf{\rho})}{\omega_{c e n t r}^{2}(\mathbf{\rho})}=2 \frac{\min _{\mathbf{k}} \omega_{n+1}^{2}(\mathbf{k}, \boldsymbol{\rho})-\max _{\mathbf{k}} \omega_{n}^{2}(\mathbf{k}, \boldsymbol{\rho})}{\min _{\mathbf{k}} \omega_{n+1}^{2}(\mathbf{k}, \boldsymbol{\rho})+\max _{\mathbf{k}} \omega_{n}^{2}(\mathbf{k}, \boldsymbol{\rho})}\right\}
$$

where $\rho$ is a vector with length equal to number of elements containing the densities of each element, and $n$ is the number of the bottom eigenvalue that defined the bandgap analyzed. 
It is important to notice that in this work, for engineering purposes, the densities in the vector $\rho=\left\{\rho_{e}, e=1, \ldots, N_{\text {elements }}\right\}$ can only assume the values of 0 or 1 . No interpolation between the two values is considered, the element could be "alive" (taken into account in the FE formulation) or "dead" (part of the air domain that is not considered in the FE formulation).

\subsection{Sensitivity analysis}

The goal is to capture how much the bandgap varies switching on/off each single element, this is a well-suited problem for the Evolutionary Structural Optimization framework, since the answer can be derived analytically [17].

It is possible to define each eigenvalue of the problem as follows:

$$
\begin{aligned}
& \omega_{n}^{2}=\frac{k_{n}}{m_{n}} \\
& k_{n}=\mathbf{u}_{n}^{T} \mathbf{K} \mathbf{u}_{n} \\
& m_{n}=\mathbf{u}_{n}^{T} \mathbf{M} \mathbf{u}_{n}
\end{aligned}
$$

where $k_{n}$ is the modal stiffness, $m_{n}$ is the modal mass and $\mathbf{u}_{n}=\mathbf{T} \tilde{\mathbf{u}}_{n}$ is the eigenvector.

The sensitivity with respect to the variables is:

$$
\frac{\partial \omega_{n}^{2}}{\partial \rho_{e}}=\frac{1}{m_{n}} \frac{\partial k_{n}}{\partial \rho_{e}}-\frac{k_{n}}{m_{n}^{2}} \frac{\partial m_{n}}{\partial \rho_{e}}=\frac{1}{m_{n}}\left(\frac{\partial k_{n}}{\partial \rho_{e}}-\omega_{n}^{2} \frac{\partial m_{n}}{\partial \rho_{e}}\right)=\frac{1}{m_{n}}\left(\frac{\partial\left(\mathbf{u}_{n}^{T} \mathbf{K} \mathbf{u}_{n}\right)}{\partial \rho_{e}}-\omega_{n}^{2} \frac{\partial\left(\mathbf{u}_{n}^{T} \mathbf{M} \mathbf{u}_{n}\right)}{\partial \rho_{e}}\right)
$$

Assuming that modifying only few elements the shape of the eigenvector will not change significantly:

$$
\frac{\partial \mathbf{u}_{n}}{\partial \rho_{e}} \approx 0
$$

equation (14) becomes:

$$
\frac{\partial \omega_{n}^{2}}{\partial \rho_{e}}=\frac{1}{m_{n}}\left(\frac{\partial\left(\mathbf{u}_{n}^{T} \mathbf{K} \mathbf{u}_{n}\right)}{\partial \rho_{e}}-\omega_{n}^{2} \frac{\partial\left(\mathbf{u}_{n}^{T} \mathbf{M} \mathbf{u}_{n}\right)}{\partial \rho_{e}}\right)=\frac{1}{m_{n}} \mathbf{u}_{n}^{T}\left(\frac{\partial \mathbf{K}}{\partial \rho_{e}}-\omega_{n}^{2} \frac{\partial \mathbf{M}}{\partial \rho_{e}}\right) \mathbf{u}_{n}
$$

from which it is clear how the change of the density of a single element can contribute to the change of the modulus of each eigenvalue by means of a combination of kinetic and potential energy.

It is possible to define a parameter representing the sensitivity obtained:

$$
\alpha_{n}^{e}=\frac{1}{m_{n}} \mathbf{u}_{n}^{T}\left(\frac{\partial \mathbf{K}}{\partial \rho_{e}}-\omega_{n}^{2} \frac{\partial \mathbf{M}}{\partial \rho_{e}}\right) \mathbf{u}_{n}
$$

By examining equation (17), it is clear that in order to increase the modulus of $\omega_{n}^{2}$ it is necessary to set:

$$
\rho_{e}=0 \text { for } e \mid \alpha_{n}^{e}>0
$$

Since the optimization problem defined in (12) involves both the upper and the lower extrema of the bandgap, it is necessary to define also the sensitivity for the upper eigenvalue of the bandgap: 


$$
\alpha_{n+1}^{e}=\frac{1}{m_{n+1}} \mathbf{u}_{n+1}^{T}\left(\frac{\partial \mathbf{K}}{\partial \rho_{e}}-\omega_{n+1}^{2} \frac{\partial \mathbf{M}}{\partial \rho_{e}}\right) \mathbf{u}_{n+1}
$$

By means of (17), (18) and (19) the sensitivity number referred to the bandgap width is:

$$
\alpha_{b g(n, n+1)}^{e}=\alpha_{n+1}^{e}-\alpha_{n}^{e}
$$

The Evolutionary Structural Optimization technique is such that the change in the density is only possible in one direction, i.e. from one phase material to the other one and not the vice versa.

A slight modification is the so-called Bidirectional ESO technique that allows both "directions" of change of the element density depending on the $\alpha_{b g(n, n+1)}^{e}$ value. In order to correctly implement the technique it is important to notice that:

$$
\begin{gathered}
\sum_{e=1}^{N_{\text {elements }}} \alpha_{n}^{e}=0 \\
\sum_{e=1}^{N_{\text {elements }}} \alpha_{n+1}^{e}=0
\end{gathered}
$$

It is important to notice that in order to obtain a feasible design the general periodic structure must not have regions with suspended material, therefore this constraint must be added to the optimization algorithm.

Another important issue regards the numerical implementation: easily these kinds of algorithms end up with checkerboard topology, and the particular implementation for solid/air phases can lead to suspended regions of material. In order to avoid such effects, a filtering subroutine is introduced in the algorithm.

\subsection{Optimization algorithm}

Following the path of reasoning introduced in the previous section, equation (18), the core of the algorithm is:

$$
\begin{gathered}
\rho_{e}=0 \text { for } e \mid \alpha_{b g(n, n+1)}^{e}>0 \text { [for elements to be "killed"] } \\
\rho_{e}=1 \text { for } e \mid \alpha_{b g(n, n+1)}^{e}<0 \text { [for elements to be "activated"] }
\end{gathered}
$$

In this work, $n$ is chosen at the beginning of the optimization procedure corresponding to the eigenvalue that defines the bottom boundary for the bandgap.

During the optimization procedure the value of $n$ may change from the starting one but the bandgap will always be centered around the desired frequency.

A general observation is mandatory in relation to (22): since the optimization concerns solid-air PnCs and the air domain is treated by eliminating the related degrees of freedom from the matrix formulation, it is not possible to define the densities in the hole. Therefore, the algorithm is built so that the densities of the elements in the material domain in the neighborhood of the hole define which elements to "live" in the hole itself.

The optimization scheme is described in the next Figure 4. 


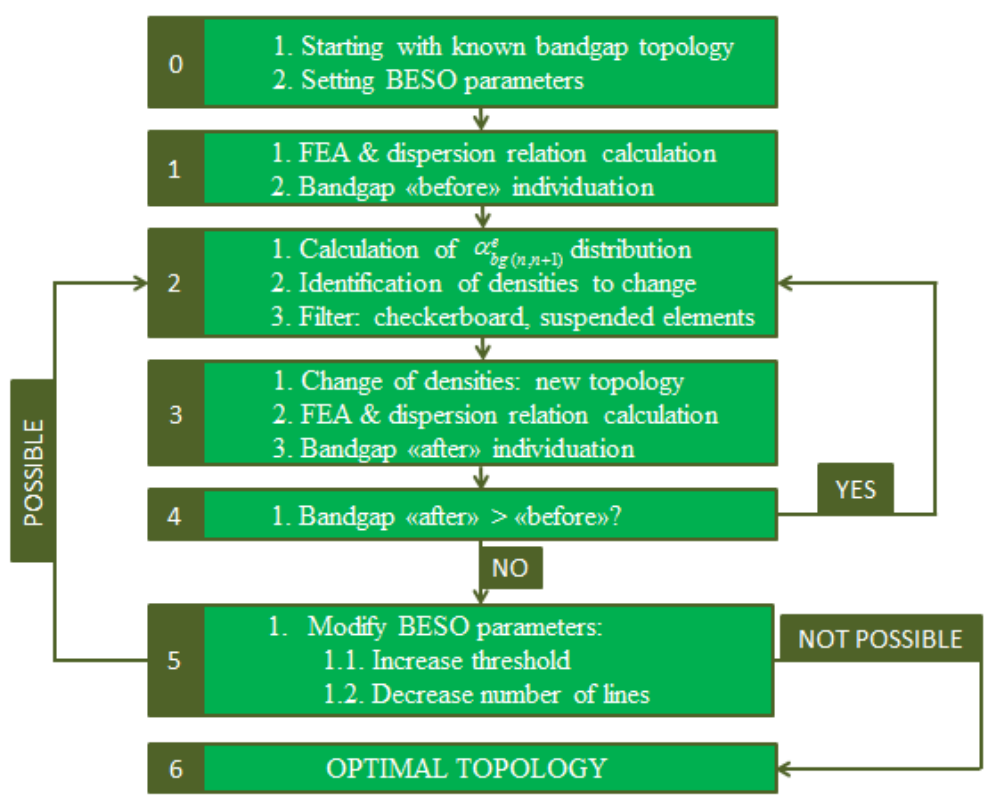

Figure 4: Optimization algorithm.

The parameters governing each BESO optimization iteration (see Figure 4) are:

- nlines ${ }^{d}$ maximum number of lines of elements near to the hole to be "killed"

- nlines ${ }^{l}$ maximum number of lines of elements in the hole to be "activated"

- nelem ${ }^{d}$ maximum number of elements to be "killed"

- nelem ${ }^{l}$ maximum number of elements to be "activated"

- $t h r^{d}$ threshold for elements to be "killed", $t h r^{d}>0$

- $t h r^{l}$ threshold for elements to be "activated", $t h r^{l}<0$

where ${ }^{d}$ stands for "dying" and ' stands for "living".

At each iteration, elements to be "killed" are:

$$
\mathbf{e}^{d}=\left\{e \mid \alpha_{b g(n, n+1)}^{e}>t h r^{d} \text {, within nlines }{ }^{d} \text { and } \text { nelem }^{d}\right\}
$$

At each iteration, elements to be "activated" are:

$$
\mathbf{e}^{l}=\left\{e_{\text {new }} \mid e_{\text {new }} \text { is near to } e, e \mid \alpha_{b g(n, n+1)}^{e}<\text { thr }^{l} \text { within } \text { nlines }^{d} \text { and } \text { nelem }^{d}, e_{\text {new }} \in \text { nlines }^{l}\right\}
$$

\section{NUMERICAL EXAMPLE}

In this section, the optimization procedure is illustrated starting from the well-known circle topology shown in Figure 5. This topology presents a complete bandgap in the 2D plane strain case. The parameters for the BESO optimization are set as:

- nlines $^{d}=2: 1$

- nlines $^{l}=2: 1$

- nelem $^{d}=400$

- nelem $^{l}=400$

- $t h r^{d}=0.20: 0.75$ normalized w.r.t. the max absolute value of the selected elements

- $t h r^{l}=-0.05 \quad$ normalized w.r.t. the max absolute value of the selected elements 


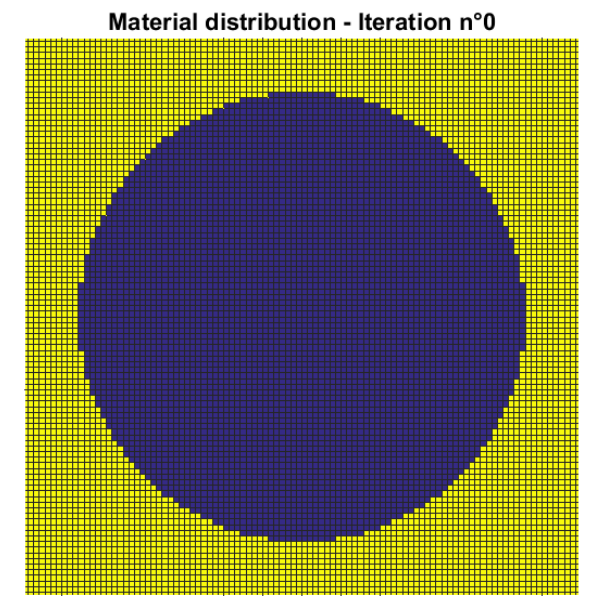

Figure 5: Material distribution for the PnC unit cell starting topology.

The hole has a circular shape of diameter $0.8 a$, with a unit cell characteristic dimension (here $a=5.5 \mu m$ ).

The unit cell is discretized in 100x100 Q4 square FE.

It is worth to notice that the optimum engineering topology is found after 13 iterations, see Figure 6. From the starting iteration, it is possible to identify the final shape following the highlighted elements that will be removed and added (see Figure 7). The bandgap in relative terms w.r.t. the center frequency increases from $14 \%$ to $89 \%$, as shown in Figure 8.

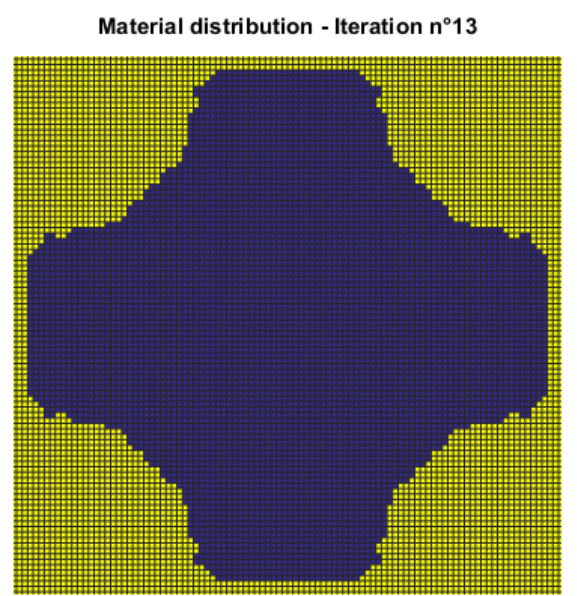

Figure 6: Material distribution for the PnC unit cell optimal topology, after 13 iterations.
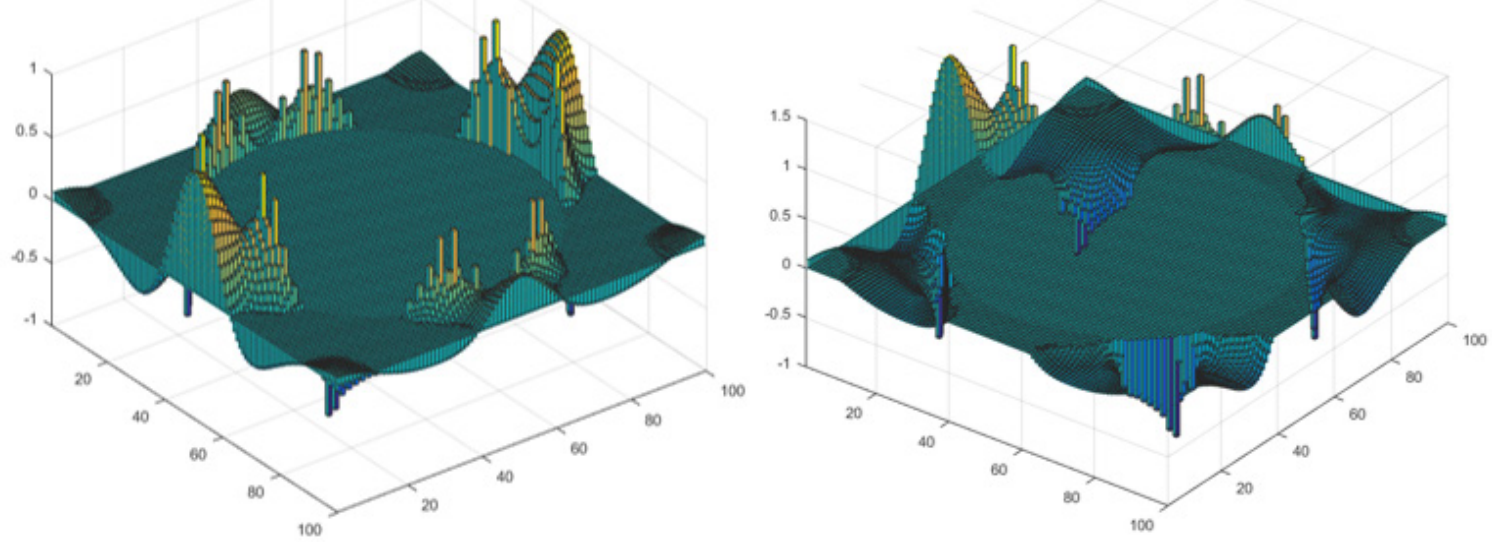

Figure 7: Distributions of $\alpha_{b g(n, n+1)}^{e}$ : dying elements on left figure, living elements on right figure. 

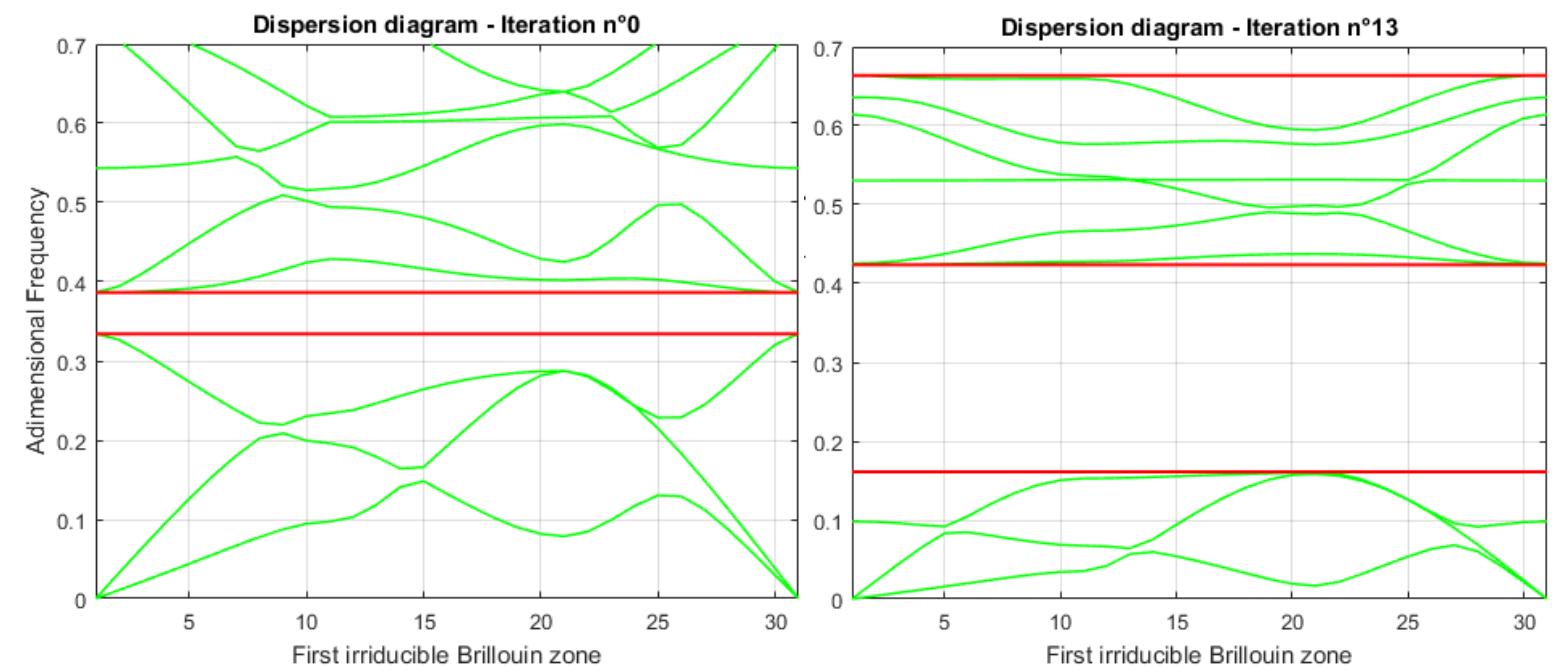

Figure 8: Comparison between dispersion relation diagrams corresponding to: starting topology (bg,rel=14\%) on the left and optimal topology (bg,rel=89\%) on the right.

The dimensionless frequency is calculated as follows:

$$
\begin{aligned}
& f_{a \mathrm{dim}}=\frac{f_{[\mathrm{Hz}]} a_{[\mu m]}}{v_{p[\mu \mathrm{m} / \mathrm{s}]}} \\
& v_{p}=\sqrt{\frac{\lambda+2 \mu}{\rho}}
\end{aligned}
$$

The optimal topology of Figure 6 can be generalized also to different materials: it is only a matter of ratios between the elastic and the mass properties. In particular, assuming that the Poisson ratio varies between 0.2 and 0.3 the only important parameter is the square root of the ratio between the elastic Young Modulus and the density.

\section{SENSITIVITY TO FABRICATION IMPRECISIONS}

An important aspect of this work is the sensitivity with respect to fabrication imprecisions: the optimal bandgap must not be too sensitive with respect to little shape variation.

In order to prove that this optimal shape is "stable", the same optimization algorithm has been used "against" the optimal shape itself, choosing the BESO parameters to simulate the fabrication imprecisions.

This choice has been done starting from the element-mesh size and then deciding how many lines of material of the optimal shape could have been removed from a fabrication imprecision, namely two lines.

This information is set as parameter in the BESO algorithm, that chooses in a couple of iterations the worst fabrication imprecision possible in order to destroy the bandgap. Figure 9 shows the results of the analyses: owing to the fabrication imprecision, the bandgap is reduced to $77 \%$, instead of its optimal value $89 \%$.

\section{CONCLUSIONS}

In this work is described a new topology optimization procedure to find the optimal bandgap for solid/air phononic crystals.

First, the formulation is described in terms of finite element method, in particular the 2D plane strain simplification. Then the optimization procedure and algorithm are introduced, 
focusing on the novelty of Bidirectional Evolutionary Structural Optimization applied to the air domain.
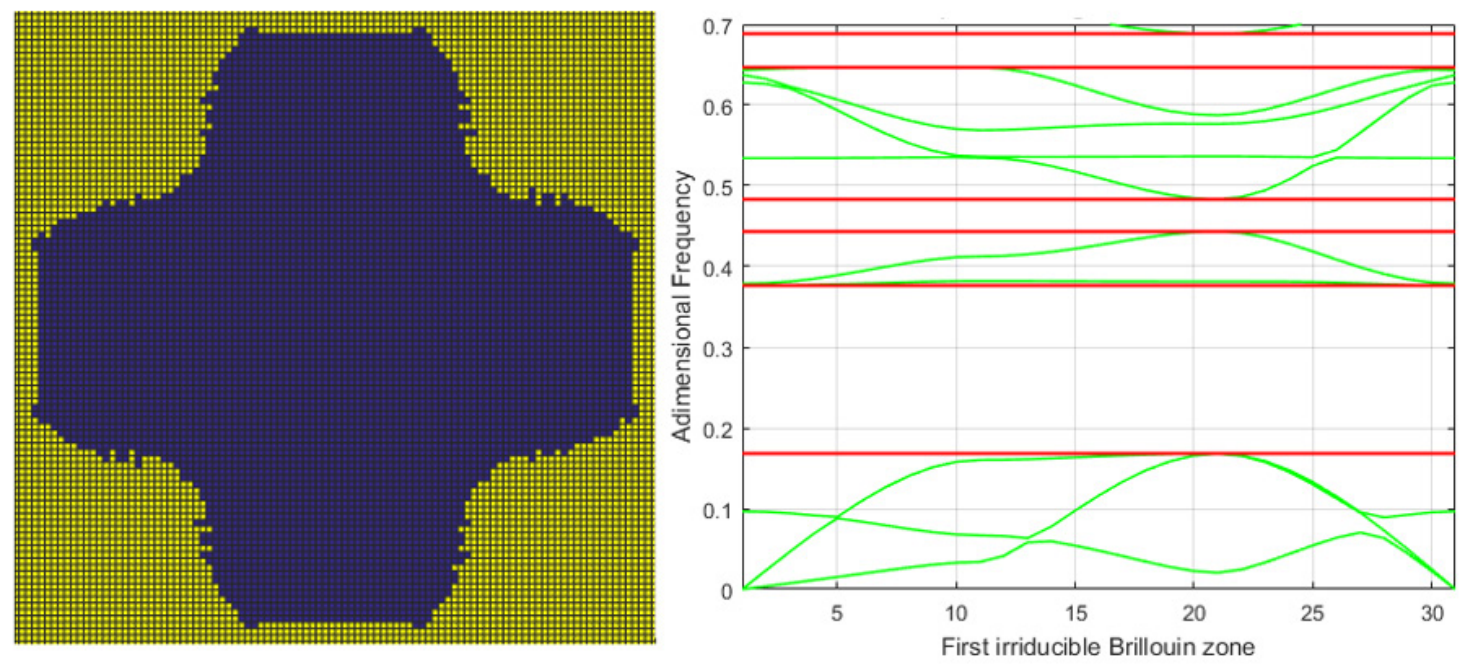

Figure 9: Results of sensitivity to fabrication process reversing the optimization algorithm: resulting topology on the left and related bandgap (bg,rel=77\%) on the right.

A numerical example is then illustrated, with starting topology defined as the well known circle-hole, that presents a bandgap of $14 \%$ and the optimal topology is derived with $90 \%$ bandgap.

This result can be extended to different materials: due to the fact that the "internal" phase is not considered the bandgap is mostly influenced by the shape of the hole and material parameter ratios.

In the last section, a sensitivity analysis is introduced in order to show that the optimal shape is "stable" with respect to fabrication imprecisions: the optimization algorithm is forced "against" the optimal topology itself in order to destroy the bandgap. The result is a decrease of the bandgap from $89 \%$ to $77 \%$, which is still a good result.

\section{REFERENCES}

[1] T. Gorishnyy, M. Maldovan, C. Ullal, and E. Thomas, "Sound ideas," Phys. World, vol. 18, no. 12, pp. 24-29, 2005.

[2] M. Maldovan, "Sound and heat revolutions in phononics.," Nature, vol. 503, no. 7475, pp. 209-17, 2013.

[3] S. Wang, L. C. Popa, and D. Weinstein, "GAN MEMS RESONATOR USING A FOLDED PHONONIC CRYSTAL STRUCTURE Massachusetts Institute of Technology , Cambridge , MA,”pp. 72-75, 2014.

[4] B. Bahr, R. Marathe, and D. Weinstein, "Phononic crystals for acoustic confinement in CMOS-MEMS resonators," IFCS 2014 - 2014 IEEE Int. Freq. Control Symp. Proc., no. iii, 2014.

[5] O. Sigmund and J. S. Jensen, "Systematic design of phononic band-gap materials and structures by topology optimization.," Philos. Trans. A. Math. Phys. Eng. Sci., vol. 361, no. 1806 , pp. 1001-1019, 2003.

[6] G. A. Gazonas, D. S. Weile, R. Wildman, and A. Mohan, "Genetic algorithm 
optimization of phononic bandgap structures," Int. J. Solids Struct., vol. 43, no. 18-19, pp. 5851-5866, 2006.

[7] O. R. Bilal and M. I. Hussein, "Optimization of phononic crystals for the simultaneous attenuation of out-of-plane and in-plane waves," ASME 2011 Int. Mech. Eng. Congr. Expo. IMECE 2011, vol. 8, pp. 969-972, 2011.

[8] H. W. Dong, X. X. Su, Y. S. Wang, and C. Zhang, "Topology optimization of twodimensional asymmetrical phononic crystals," Phys. Lett. Sect. A Gen. At. Solid State Phys., vol. 378, no. 4, pp. 434-441, 2014.

[9] F. Meng, X. Huang, and B. Jia, "Bi-directional evolutionary optimization for photonic band gap structures," J. Comput. Phys., vol. 302, pp. 393-404, 2015.

[10] M. Collet, M. Ouisse, M. Ruzzene, and M. N. Ichchou, "Floquet-Bloch decomposition for the computation of dispersion of two-dimensional periodic, damped mechanical systems," Int. J. Solids Struct., vol. 48, no. 20, pp. 2837-2848, 2011.

[11] M. I. Hussein, "Reduced Bloch Mode Expansion for Periodic Media Band Structure Calculations," Proc. Math. Phys. Eng. Sci., vol. 465, no. 2109, pp. 2825-2848, 2009.

[12] J. H. Sun and T. T. Wu, "Propagation of acoustic waves in phononic-crystal plates and waveguides using a finite-difference time-domain method," Phys. Rev. B - Condens. Matter Mater. Phys., vol. 76, no. 10, pp. 1-8, 2007.

[13] A. S. Phani, J. Woodhouse, and N. A. Fleck, "Wave propagation in two-dimensional periodic lattices," J. Acoust. Soc. Am., vol. 119, no. 4, p. 1995, 2006.

[14] Y.-F. Wang, Y.-S. Wang, and X.-X. Su, "Large bandgaps of two-dimensional phononic crystals with cross-like holes," J. Appl. Phys., vol. 110, no. 11, p. 113520, 2011.

[15] A. R. Diaz, A. G. Haddow, and L. Ma, "Design of band-gap grid structures," Struct. Multidiscip. Optim., vol. 29, no. 6, pp. 418-431, 2005.

[16] L. Brillouin, Wave Propagation in Periodic Structures. New York, 1953.

[17] Y. M. Xie and G. P. Steven, Evolutionary Structural Optimization. 1997. 